\title{
Extending the Velocity-dependent One-scale String Evolution Model
}

\author{
C. J. A. P. Martins * and E. P. S. Shellard ${ }^{\dagger}$ \\ Department of Applied Mathematics and Theoretical Physics \\ Centre for Mathematical Sciences, University of Cambridge \\ Wilberforce Road, Cambridge CB3 OWA, U.K.
}

(March 30, 2000)

\begin{abstract}
We provide a general overview of the velocity-dependent one-scale model for cosmic string evolution and discuss two further extensions to it. We introduce and justify a new ansatz for the momentum parameter $k$, and also incorporate the effect of radiation backreaction. We thus discuss the evolution of the basic large-scale features of cosmic string networks in all relevant cosmological scenarios, concentrating in particular on the 'scaling' solutions relevant for each case. In a companion paper, we show, by comparing with numerical simulations, that this model provides an accurate description of the large-scale features of cosmic string networks.
\end{abstract}

\section{INTRODUCTION}

The velocity-dependent one-scale (VOS) model provides the most convenient and reliable method by which to calculate the large-scale quantitative properties of a string network in cosmological and other contexts [1 [- 3. It is widely used for making quantitative predict ions of the potential observational implications of cosmic strings 任. Given its simplicity, it is remarkable how well the VOS model performs when tested against high resolution numerical simulations of string networks [5]. It is well-known that string evolution is a complex physical process with a build-up of small-scale structure on the strings, which is very computationally demanding to model accurately [6]. Analytic approaches like the VOS model, abandon the possibility of describing the statistical physics of the string network accurately and concentrate instead on its thermodynamics. In other words, a small number of macroscopic quantities are selected and the microscopic string equations of motion are used to derive evolution equations for these averaged quantities. The price to be paid in this approach is that the averaging process introduces phenomenological parameters whose values are not specified by the model itself. Instead, one must still fix these parameters by direct comparison with numerical simulations.

The VOS model is a generalization of the 'one-scale' model pioneered by Kibble [9] (see also ref. [10]) which describes string motion in terms of a single correlation length $L$. By incorporating a variable rms velocity $v$, the VOS model extends its validity into early regimes with frictional damping and across the important matter-radiation transition, thus giving a quantitative picture of the complete history of a cosmic string network. Other analytic approaches to string evolution have attempted to incorporate the additional small-scale structure seen in numerical simulations. This includes a 'kink-counting' model [11], a functional approach [12], a 'three-scale' model [13] and a 'wiggly' model [14]. While these are important for characterising detailed network features, they introduce a significant number of further phenomenological parameters which must be fixed by simulations (and which remain rather uncertain). Nevertheless, for describing the large-scale properties of a long-string network, the VOS model has proved to be sufficient for a good quantitative fit using only a single parameter, the loop chopping efficiency $\tilde{c}$.

The purpose of the present paper is, first, to provide a concise exposition of the VOS string evolution model. We summarise how it can be applied to describe cosmological string evolution, including late times with a cosmological constant, and we present the very different histories of both GUT- and electroweak-scale strings. Secondly, we propose an improvement of the VOS model by presenting a new ansatz for the momentum parameter $k$, which we justify both analytically and numerically. Thirdly, we present a further extension incorporating radiation backreaction, which provides small corrections to the cosmological scaling laws and which also compares favourably with published results of global string simulations. Finally, we review generalizations in a curved FRW spacetime, giving some further asymptotic scaling solutions. We report on detailed comparisons between numerical string simulations and the VOS model elsewhere $[$, 15,16$]$.

${ }^{*}$ Also at C. A. U. P., Rua das Estrelas s/n, 4150 Porto, Portugal. Electronic address: C.J.A.P.Martins @ damtp.cam.ac.uk

${ }^{\dagger}$ Electronic address: E.P.S.Shellard @ damtp.cam.ac.uk 


\section{THE VOS STRING NETWORK MODEL}

The velocity-dependent one-scale model has been described in considerable detail elsewhere [1, 3, 17, 19, so here we limit ourselves to a brief summary which highlights the features that will be important for what follows. Also for simplicity, we will only discuss the evolution of the long string network, even though this formalism is also applicable to the loop population, mutatis mutandis. We will discuss this case in detail elsewhere [16].

\section{A. The averaged evolution equations} by

Averaged quantities which we could use to describe the string network are its energy $E$ and RMS velocity $v$ defined

$$
E=\mu a(\tau) \int \epsilon d \sigma, \quad v^{2}=\frac{\int \dot{\mathbf{x}}^{2} \epsilon d \sigma}{\int \epsilon d \sigma}
$$

where the string trajectory $\mathbf{x}(\sigma, t)$ is parametrised by the worldsheet coordinates $\sigma$ and $t$ and the 'energy density' $\epsilon(\sigma, t)$ gives the string length per unit $\sigma$ along the string.

Any string network divides fairly neatly into two distinct populations, long (or 'infinite') strings and small closed loops with corresponding quantities denoted by a subscript $\infty$ and $\ell$ respectively. The long string network is a Brownian random walk on large scales and can be characterised by a correlation length $L$. This can be used to replace the energy $E_{\infty}=\rho_{\infty} V$ in long strings in our averaged description, that is,

$$
\rho_{\infty} \equiv \frac{\mu}{L^{2}}
$$

A phenomenological term must then be included to account for the loss of energy from long strings by the production of loops, which are much smaller than $L$. A 'loop chopping efficiency' parameter $\tilde{c}$ is introduced to characterise this loop production as

$$
\left(\frac{d \rho_{\infty}}{d t}\right)_{\text {to loops }}=\tilde{c} v_{\infty} \frac{\rho_{\infty}}{L}
$$

In this approximation, we would expect the loop parameter $\tilde{c}$ to remain constant irrespective of the cosmic regime, because it is multiplied by factors which determine the string network self-interaction rate.

From the microscopic string equations of motion, one can then average to derive the evolution equation for the correlation length $L$,

$$
2 \frac{d L}{d t}=2 H L\left(1+v_{\infty}^{2}\right)+\frac{L}{\ell_{\mathrm{f}}} v_{\infty}^{2}+\tilde{c} v_{\infty}
$$

where $H$ is the Hubble parameter and $\ell_{\mathrm{f}}$ is a friction damping length scale. The first term in (2.3) is due to the stretching of the network by the Hubble expansion which is modulated by the redshifting of the string velocity. The second term is due to frictional interactions by a high density of background particles scattering off the strings. The friction length scale $\ell_{\mathrm{f}}$ (defined in ref. [1]) typically depends on the background temperature as $\ell_{\mathrm{f}} \approx \imath^{2}(1) \mu T^{-3}$, so that it grows with the scale factor as $a^{3}$. It usually becomes irrelevant after a time $t_{*} \approx(G \mu)^{-1} t_{c}$ (with $t_{c}$ being the epoch at which the network was formed) which is a very short time for GUT-scale strings but can be as late as $t_{0}$ for electroweak strings. Note also that equation (2.3) is valid for an arbitrary flat FRW model with $H$ given by the Friedmann equation,

$$
H^{2} \equiv\left(\frac{\dot{a}}{a}\right)^{2}=H_{0}^{2}\left(\Omega_{\mathrm{R} 0} a^{-4}+\Omega_{\mathrm{M} 0} a^{-3}\right)+\frac{1}{3} \Lambda .
$$

where $\Omega_{\mathrm{R} 0}$ and $\Omega_{\mathrm{M} 0}$ are the fractional radiation and matter densities today at $t_{0}, \Lambda$ is the cosmological constant and we take $a\left(t_{0}\right)=1$.

One can also derive an evolution equation for the long string velocity with only a little more than Newton's second law

$$
\frac{d v_{\infty}}{d t}=\left(1-v_{\infty}^{2}\right)\left[\frac{k}{L}-\left(2 H+\frac{1}{\ell_{\mathrm{f}}}\right) v_{\infty}\right]
$$


where $k$ is called the 'momentum parameter'. The first term is the acceleration due to the curvature of the strings and the second damping term is from both the expansion and background friction. The parameter $k$ is defined by

$$
k \equiv \frac{\left\langle\left(1-\dot{\mathbf{x}}^{2}\right)(\dot{\mathbf{x}} \cdot \mathbf{u})\right\rangle}{v\left(1-v^{2}\right)},
$$

with $\dot{\mathbf{x}}$ the microscopic string velocity and $\mathbf{u}$ a unit vector parallel to the curvature radius vector. In previous work 11.2.18.3, we left $k$ as a second phenomenological parameter, while pointing out that it is related to small-scale structure and also demonstrating specified asymptotic dependencies on the velocity. In the next section, however, we justify an accurate ansatz for $k$ which removes this additional freedom. For most relativistic regimes relevant to cosmic strings it is sufficient to define it as follows:

$$
k_{\mathrm{rel}}(v)=\frac{2 \sqrt{2}}{\pi} \frac{1-8 v^{6}}{1+8 v^{6}} .
$$

In the extreme friction-dominated case $(v \rightarrow 0)$, we have the nonrelativistic limit $k_{\mathrm{nr}}=2 \sqrt{2} / \pi$ with a more complicated ansatz than (2.7) interpolating between these limits for intermediate regimes.

Finally, we end this summary by noting that the VOS model has been extensively compared with the results of numerical simulations [7. 8. [. 16] and shown to provide a good fit to the large-scale properties of a string network. In particular, it matches well the evolution between asymptotic regimes as a network passes through the matterradiation transition. Comparisons with numerical simulations confirm the constancy of the only free parameter, the loop chopping efficiency $\tilde{c}$, and fix its value to be [5]

$$
\tilde{c}=0.23 \pm 0.04 \text {. }
$$

The VOS model for any flat FRW cosmology, then, consists of the evolution equations (2.3) and (2.5) with the parameters $c$ specified in (2.8) and $k$ given by (2.7) (or a more accurate general expression given below) and the scale factor $a$ satisfying the Friedmann equation (2.4).

\section{B. Scale-invariant solutions}

We now start to use the VOS model to provide a general overview of the evolution of string networks in various cosmological scenarios. First we analyse some basic late-time properties of cosmic string networks, neglecting the effect of friction due to particle scattering. A crucial question is whether or not they can reach a 'scale invariant' attractor solution which is required, among other things, for a Harrison-Zel'dovich spectrum of primordial density fluctuations to be generated. This can be discussed by analysing the VOS equations (2.3) and (2.5).

Scale-invariant solutions of the form $L \propto t, L \propto H^{-1}$ or $L \propto d_{H}$, together with $v_{\infty}=$ const., only appear to exist when the scale factor is a power law of the form

$$
a(t) \propto t^{\beta}, \quad \beta=\text { const. }, \quad 0<\beta<1 .
$$

This condition implies that

$$
L \propto t \propto H^{-1} \propto d_{H},
$$

with the proportionality factors dependent on $\beta$. It is useful to introduce the following useful parameters to describe the relative correlation length and densities, defining them respectively as

$$
L=\gamma t, \quad \zeta \equiv \gamma^{-2}=\rho_{\infty} t^{2} / \mu .
$$

By looking for stable fixed points in the VOS equations, we can express the actual scaling solutions in the following implicit form:

$$
\gamma^{2}=\frac{k(k+\tilde{c})}{4 \beta(1-\beta)}, \quad v^{2}=\frac{k(1-\beta)}{\beta(k+\tilde{c})},
$$

where $k$ is the constant value of $k(v)$ given by solving the second (implicit) equation for the velocity. Although it may not be obvious by inspection, it is easy to verify numerically that this solution is well-behaved and stable for all realistic parameter values. 
If the scale factor is not a power law, then simple scale-invariant solutions like (2.12) do not exist. Physically this happens because the network dynamics are unable to adapt rapidly enough to the changes in the background cosmology. A prime example of this is, of course, the transition between the radiation and matter-dominated eras. The evolution of a GUT-scale network shown in fig. 1 illustrates asymptotic regimes in which string evolution is scale-invariant, as well as the matter-radiation transition where it is not. Note that for realistic cosmological parameter choices, a string network today is still only slowly approaching its asymptotic matter density. Since, the cosmological importance of the changes in the network properties during the matter-radiation transition cannot be over-emphasised, it is important to calculate these accurately either with direct numerical simulations or with the VOS or similar analytic model. The same is also true for late time curvature or cosmological constant domination.

\section{Friction-dominated scaling solutions}

During friction-dominated epochs one has different 'scaling' solutions. However, these are no longer 'scale-invariant', since in this case the network retains a memory of its initial conditions, and in particular of the epoch of formation. This can be conveniently expressed by a parameter

$$
\theta \sim\left(\frac{t_{c}}{t_{P l}}\right)^{1 / 2}
$$

which is essentially the value of the ratio of the damping terms due to friction and Hubble damping, measured at the epoch of string formation.

Thus in realistic cosmological contexts one can have two different regimes [1]3]. The first is a 'stretching' regime,

$$
\frac{L}{L_{c}}=\left(\frac{t}{t_{c}}\right)^{1 / 2}, \quad v=\frac{t}{\theta L_{c}}
$$

which is an early-time, transient period which will occur when the initial string density and velocity are sufficiently low - for example, as a result of a slow first-order phase transition. In this case the network starts out with a correlation length significantly larger than the damping length and so is 'frozen', and is conformally stretched. However the damping length is growing as $\ell_{f} \propto t^{3 / 2}$, so it quickly catches up with it, ending this regime. However, this can last for many orders of magnitude in time for electroweak-scale networks. Although this is not cosmologically relevant except for extremely light strings, the analogous regime in the matter-dominated case would be

$$
L \propto t^{2 / 3}, \quad v \propto t^{4 / 3} .
$$

The true attractor solution for a friction-dominated epoch, which follows the stretching regime (if this exists) is the Kibble regime, which in the radiation era has the form

$$
\frac{L}{L_{c}}=\left[\frac{2 k_{n r}\left(\tilde{c}+k_{n r}\right)}{3 \theta}\right]^{1 / 2}\left(\frac{t}{t_{c}}\right)^{5 / 4}, \quad v=\left[\frac{3 k_{n r}}{2 \theta\left(\tilde{c}+k_{n r}\right)}\right]^{1 / 2}\left(\frac{t}{t_{c}}\right)^{1 / 4},
$$

where $k_{n r}$ is the value of the momentum parameter in the nonrelativistic limit given above. In this case the correlation length stays halfway between the damping length and the horizon length. Again there is a matter era analogue, which as the form

$$
L \propto t^{3 / 2}, \quad v \propto t^{1 / 2},
$$

but this is rarely relevant cosmologically. In the extreme case of an electroweak scale network, friction domination ends after radiation-matter equality, but not far enough away from it for this regime to be reached.

These points are illustrated in fig. 2 2 where we see the evolution of an electroweak-scale string network. It is interesting to note the scaling behaviour for two cases with very different initial conditions from extreme first- and second-order transitions. The high density strings from a second-order transition quickly approach the Kibble scaling regime (2.16) discussed above. However, the low density strings from a first-order transition begin in a distinct stretching regime (2.14) and persist in it with their density falling slowly until it matches that for the attractor Kibble regime. In this case, the string network retains a 'memory' of its initial density for about ten orders of magnitude in cosmic time. However, even during the friction-dominated regime the network is able to erase this memory once the Kibble regime is reached. On the other hand, the memory of the epoch of formation is not erased - one could in principle recover it by measuring the parameter $\theta$. This can also be seen for GUT strings in fig. 1, although the 
network does not have time to relax into a definite scaling regime before friction-domination ends. For electroweak strings, there are also interesting departures from scaling behaviour at the matter-radiation transition and the network remains friction-dominated until about three orders of magnitude in time afterwards. Also note that in the $\Omega_{m}=1$ case the strings only reach the relativistic regime at about the present time, and in the observationally preferred case $\Omega_{m}=0.2, \Omega_{\Lambda}=0.8$ they are always non-relativistic. This point is crucial, among other things, for a quantitative analysis of the evolution of superconducting strings and vortons [20,21].

\section{A cosmological constant}

We can also use the VOS model in a flat background to discuss the domination at late times by a cosmological constant $\Lambda$ [22,19], a model for which there appears to be growing observational evidence. In the extreme asymptotic case when the universe is inflating we have $a \propto \exp (H t)$ with $H=\sqrt{\Lambda / 3}$. The network will 'freeze out' and will simply be conformally stretched, that is,

$$
L \propto a, \quad v_{\infty} \propto a^{-1}
$$

where, as soon as the strings become nonrelativistic $k_{\mathrm{nr}}=2 \sqrt{2} / \pi$, their product satisfies

$$
L v_{\infty}=\frac{2 \sqrt{2}}{\pi} H .
$$

Of course, this solution will only apply at early times actually during inflation. At the present time we will only be slowly approaching a new stretching regime, so we have to solve the VOS model explicitly. This is shown for a model in which $\Omega_{\Lambda}=0.8$, as a dashed line at late times in figs. 112, as well as in detail for GUT-scale strings in fig. 3 . It is clear that there is a significant fall in the string density and velocity, an effect which, for example, would affect the large-angle anisotropies in the cosmic microwave sky. The evolution of the string network is clearly not scale-invariant during any period after $t_{\text {eq }}$.

\section{THE MOMENTUM PARAMETER}

Having introduced the VOS model and some of its key cosmological implications, we now turn to a more detailed discussion of the so-called 'momentum parameter' $k$ defined in (2.6) and which is important for solving (2.5). String velocities are determined by the current acceleration to which they are subjected from the local string curvature, as well as their 'bulk' momentum left-over from previous accelerations. Heuristically, we can imagine separating the velocity into these the curvature 'c' and bulk 'p' contributions as $\dot{\mathbf{x}}=\dot{\mathbf{x}}_{\mathrm{c}}+\dot{\mathbf{x}}_{\mathrm{p}}$. In the extreme friction-dominated limit, the velocity is entirely due to the curvature and reaches a limiting average velocity set by the friction length scale, $v_{\mathrm{c}} \equiv\left\langle\dot{\mathbf{x}}_{\mathrm{c}}^{2}\right\rangle^{1 / 2}=\ell_{f} / L$. However, as the velocity increases towards relativistic values we can expect the momentum contribution to become larger. Let us suppose that their relative contribution is proportional to some power law of the total velocity $v$, that is, $v_{\mathrm{p}} / v_{\mathrm{c}} \propto v^{\alpha}$ where $\alpha$ is clearly greater than unity. In this case, one can find after some straightforward (although tedious) manipulations that the following approximate relation holds for the momentum parameter $k$

$$
k \sim \frac{1-2^{\alpha} v^{2 \alpha}}{1+2^{\alpha} v^{2 \alpha}} .
$$

In this we have also used the fact that flat spacetime analytic calculations [2, 3] have shown that

$$
k(1 / \sqrt{2})=0
$$

which holds exactly. Note that the above expression means that (3.1) can also be approximately written as

$$
k \sim \frac{1-\left(v_{p} / v_{c}\right)^{2}}{1+\left(v_{p} / v_{c}\right)^{2}} .
$$

We can determine $\alpha$ by studying the well-known 23] helicoidal string solution in flat space but perturbed by a frictional force. This solution is 


$$
\mathbf{x}=\left(A \sin \sigma(\cos t+\eta), A \cos \sigma(\cos t+\eta), \sqrt{1-A^{2}} \sigma\right)
$$

where $0 \leq A \leq 1$ and $\eta$ is a small perturbation, which vanishes if there is no friction. Here, $A=1$ corresponds to a circular loop, while $A=0$ is a static straight string. The evolution equation for the perturbation $\eta$ has the form

$$
\ddot{\eta}+2 \dot{\eta} \frac{\sin t \cos t}{1-A^{2} \sin ^{2} t}+\eta \frac{\left(1-A^{2}\right) \sin ^{2} t-\cos ^{2} t}{1-A^{2} \sin ^{2} t}=\frac{\sin t}{\ell_{f}}\left(1-A^{2} \sin ^{2} t\right),
$$

where $\ell_{f}$ is the friction length scale. This can then be solved numerically, and from this solution one can calculate $k$. By changing parameters $A$ and $\ell_{f}$ one can do this for a wide range of velocities, and hence obtain a plot of $k=k(v)$. This is plotted in Fig. 4 and compared with cases $\alpha=2$ and $\alpha=3$ of our ansatz (3.1). It can be seen that $\alpha=3$ provides an excellent approximation in this regime.

There is, however, one problem with this simple ansatz, namely that it would give $k(0)=1$. Even though one might naively expect this to be the correct limit, it is not so. This can be seen easily as follows. Assume that the velocity of say a loop is determined only by curvature, that is, neglect the momentum contribution. (This should be valid in the non-relativistic case.) Then $k$ will be approximately given by

$$
k \approx \frac{<\left|\dot{\mathbf{x}}^{2}\right|\left(1-\dot{\mathbf{x}}^{2}\right)>}{v\left(1-v^{2}\right)} .
$$

This quantity can be easily calculated for the analytic helicoidal solution, yielding

$$
k=\frac{2 \sqrt{2}}{\pi} \frac{1-2 A^{2} / 3}{1-A^{2} / 2} .
$$

Hence we find in the small amplitude limit $A \rightarrow 0$ that

$$
k_{\mathrm{nr}}=\frac{2 \sqrt{2}}{\pi} \approx 0.9 .
$$

We also note in passing that in the relativistic limit $A \rightarrow 1$ this same calculation would give $k \approx 4 \sqrt{2}(3 / \pi) \approx 0.6$ instead of the true value $k=0$, which clearly demonstrates the importance of momentum in the relativistic case.

The final issue to be considered is the transition between the two regimes. The only reliable way of studying this issue is through direct measurement in a string network simulations with ultra-high resolution. We shall report on the details of this elsewhere [16]. Here we simply point out that we do confirm the value $k_{\mathrm{nr}}$ as the non-relativistic limit. It is then easy to find a fitting function for the transition between the regimes which has the correct asymptotic limits described previously, that is,

$$
k(v)=\frac{2 \sqrt{2}}{\pi}\left(1-v^{2}\right)\left(1+2 \sqrt{2} v^{3}\right) \frac{1-8 v^{6}}{1+8 v^{6}} .
$$

The additional factors are required to reproduce both the relativistic and non-relativistic limits accurately. Note that if one is only interested in the relativistic regime (say for GUT-scale cosmic strings, as in the present paper) then the simpler expression (2.7), that is

$$
k_{\mathrm{rel}}(v)=\frac{2 \sqrt{2}}{\pi} \frac{1-8 v^{6}}{1+8 v^{6}}
$$

should be sufficiently accurate to provide reliable results. On the other hand, a reliable approximation for small non-relativistic velocities in the friction-dominated limit extends (3.8) as

$$
k_{\mathrm{nr}}(v)=\frac{2 \sqrt{2}}{\pi}\left(1-v^{2}\right) .
$$

We plot these three ansatze in fig. 5, and also confirm the validity of $k_{\text {rel }}$ and $k_{\text {nr }}$ in the appropriate limits.

Before we end this section, however, it is wise to discuss the interpretation of parameter $k$ in this model. It should be kept in mind that this is, ab initio, a phenomenological parameter, which accounts for a number of non-trivial effects related to the presence of small-scale structures on the strings. By construction, our model does not explicitly account for these small-scale effects, and hence they end up somehow encoded in $k$. One should not therefore infer too much from the aesthetic qualities of the function $k(v)$ we find-it is simply a phenomenological parameter that does a good job. Presumably this parameter will have a much clearer interpretation in the context of a proper wiggly string evolution model [24,14]. 


\section{THE EFFECT OF RADIATION BACK-REACTION}

We now turn to some further extensions of the VOS model. The effect of gravitational back-reaction on the longstring network [11,13 can be included in the evolution equation for the correlation length (2.3) in the same way as previously achieved for the evolution of the length of a string loop [2]. For gravitational radiation the following term can be added to the right-hand side of (2.3)

$$
2\left(\frac{d L}{d t}\right)_{\mathrm{gr}} \equiv 8 \Sigma_{\mathrm{gr}} v_{\infty}^{6}=8 \tilde{\Gamma} G \mu v_{\infty}^{6}
$$

Here, $\tilde{\Gamma}$ is a constant which is a long-string analogue of the $\Gamma \approx 65$ found for the radiative decay of strings. Of course, $\tilde{\Gamma}$ will be affected by a number of physical factors such as the presence of small-scale structure, but we can expect it to satisfy $\tilde{\Gamma} \lesssim \Gamma$ and it would be surprising if it were very much smaller. Clearly, due to the high velocity power $\left(v^{6}\right)$ involved in radiative backreaction, this term will not be important in any regime where string motion is strongly friction-dominated (and hence non-relativistic). We note also that there is an interesting coincidence in the ansatz (2.7) for the momentum parameter $k$ which too has a $v^{6}$ power, but we are unsure as yet whether this has any deeper significance.

For global string radiation into Goldstone bosons or axions, the corresponding radiative decay term at a time $t$ will be

$$
2\left(\frac{d L}{d t}\right)_{\mathrm{ax}} \equiv 8 \Sigma_{\mathrm{ax}} v_{\infty}^{6}=\frac{8 \tilde{\Gamma} v_{\infty}^{6}}{2 \pi \ln (t / \delta)},
$$

where the logarithmic term arises because of the long-range fields of the global string and $\delta$ is the string width. For cosmological GUT-scale strings, the backreaction term for local strings is $\Gamma G \mu \sim 10^{-4}$ whereas for global strings it is about three orders of magnitude larger.

Note that the velocity equation has no correction at this order due to the gravitational back-reaction effects. Such effects are already included through the string curvature, which acts as a source for the velocity equation (i.e., the $1 / L$ term), which will be different in this case.

Remarkably, the inclusion of the back-reaction term does not affect the existence of a scale-invariant attractor solution. However, it does of course influence the quantitative values of the scaling parameters, as well as the timescale necessary for this solution to be reached. For example, the inclusion of back-reaction can make the approach to scaling much faster.

If the gravitational back-reaction is non-zero, one can distinguish two asymptotic cases. Firstly, if $\Sigma$ is small (of order unity at most) then the effect of back-reaction on the scaling solution will also be small. This will be the case, for example, for most local or global string networks in a cosmological context. We can express this as

$$
\gamma^{2} \approx \gamma_{0}^{2}(1+\Delta), \quad v^{2} \approx v_{0}^{2}(1-\Delta),
$$

where $\gamma_{0}$ and $v_{0}$ are the "unperturbed" scaling values, given by eqns. (2.12), and the back-reaction correction has the form

$$
\Delta=8 \beta v_{0}^{5} \Sigma=8 \beta\left[\frac{k(1-\beta)}{\beta(k+\tilde{c})}\right]^{5 / 2} \Sigma .
$$

On the other hand, for large enough values of $\Sigma$, the back-reaction term will dominate the evolution equation for the string length scale $L$, and the attractor scale-invariant solution has a different form altogether. It is not possible to write this solution in closed form, even expressing $k$ implicitly as above. However, it is possible to write it as a series. The dominant term and the first correction take the form

$$
\begin{gathered}
\gamma=\frac{k}{2 \beta}\left[\frac{8 \beta \Sigma}{k(1-\beta)}\right]^{1 / 7}\left(1+\Delta_{1}+\ldots\right), \\
v=\left[\frac{k(1-\beta)}{8 \beta \Sigma}\right]^{1 / 7}\left(1-\Delta_{1}+\ldots\right),
\end{gathered}
$$

with 


$$
\Delta_{1}=\frac{1}{2^{6 / 7} 7}(k+\tilde{c})\left[\frac{\beta}{k(1-\beta)}\right]^{5 / 7} \Sigma^{-2 / 7} .
$$

In fig. 6 we plot the approach to scaling of some relevant string networks in the radiation and matter eras. The different timescales for convergence towards the attractor solution are clearly noticeable. Here, we have chosen initial conditions that would correspond to somewhat extreme first and second order phase transitions. Also note that for the radiation era we have neglected the effect of friction due to particle scattering, in order to reproduce the initial conditions often used in numerical simulations of string networks. For each of the cases above, three curves are plotted, corresponding to the values $\Sigma=0$ (no back-reaction), $\Sigma=1.25$ (close to the maximum value that can be accurately described by the scaling solution 4.34 .4 ) and $\Sigma=5.5$ (beyond which the scaling solution (4.5 4.7) becomes accurate).

For large $\Sigma$, the effects of back-reaction seen in fig. 6 on could be quite dramatic for the string network density, but note that they are much less drastic for the string velocities. In particular, we emphasize that gravitational back-reaction alone does not slow down a string network to non-relativistic speeds - only a friction-dominated regime can achieve this.

Interestingly, there has been recent work on numerical simulations of global string networks [25] which explore the strong backreaction regime described by (4.5 4.7). These authors report a surprisingly low string density relative to the gauged case. For their expanding universe simulations in the realistic case with periodic boundary conditions, they find the following radiation and matter era densities respectively,

$$
\zeta_{\text {rad }}=0.9 \pm 0.1, \quad \zeta_{\text {mat }}=0.5 \pm 0.1
$$

These results are perfectly consistent (within the estimated error bars) with our extended VOS model if we adopt a back-reaction parameter

$$
\Sigma_{\text {ax-sim }} \approx 3
$$

Indeed, this corresponds to the approximate average value for $\Sigma_{\mathrm{ax}}$ that one would estimate for simulations of this resolution. Present limitations on numerical dynamic range give the upper bound $\ln (t / \delta) \lesssim 5$ (at the end of the simulation), implying $\Sigma_{\text {ax-sim }} \gtrsim 2$ throughout.

It is important to note, however, that the immediate extrapolation of these results (4.8) to a cosmological context would be erroneous. For example, for GUT-scale global strings, at the present time we can expect $\ln (t / \delta) \gtrsim 100$, which implies the appropriate backreaction parameter in this case will be $\Sigma_{\mathrm{ax}} \lesssim 0.1$. Such cosmic global strings are firmly in the regime (4.3 4.4) where backreaction effects are small and, in this case, these would reduce the local string density by less than 10\%. We make a more detailed comparison with [25] elsewhere [5].

\section{STRING NETWORKS IN GENERAL FRW SPACETIMES}

In this section we discuss the behaviour of our model in more general FRW universes, and in particular in open universes 17, 3, 18,22].

The evolution equations will obviously be affected by the different behaviour of the scale factor, as given by the Friedmann equation

$$
H^{2} \equiv\left(\frac{\dot{a}}{a}\right)=H_{0}^{2}\left(\Omega_{\mathrm{R} 0} a^{-4}+\Omega_{\mathrm{M} 0} a^{-3}+\Omega_{\mathrm{Q} 0} a^{-m}\right)+\frac{1}{3} \Lambda-K a^{-2} .
$$

Note that we are allowing for curvature, and also for an extra fluid whose energy density decays as $a^{-m}$. It should be kept in mind that the Friedman equation should, in general, contain a contribution for the string density, since it is possible that this becomes cosmologically important.

However, apart from these effects, one must also include an additional correction due to the curvature [18, 3]. One should note that this is essentially the curvature radius of the strings, $L$, divided by the radius of spatial curvature of the universe,

$$
\mathcal{R}=\frac{H^{-1}}{|1-\Omega|^{1 / 2}}
$$

Indeed, after a certain amount of algebra, one finds correction terms that are of the form 


$$
w=1-(1-\Omega)(H L)^{2} .
$$

Note that $\Omega$ denotes the total density of the universe. For a universe with a critical density, $\Omega=1$, we have $w=1$.

The evolution equation for the correlation length $L$ now takes the form

$$
2 \frac{d L}{d t}=2 H L+\frac{L}{\ell_{d}} \frac{v_{\infty}^{2}}{w^{2}}+\tilde{c} v_{\infty}
$$

For simplicity wa have also defined a damping length, including both the effects of Hubble damping and friction,

$$
\frac{1}{\ell_{d}}=2 H+\frac{1}{\ell_{f}}
$$

with the friction length scale $\ell_{f}$ being defined in [1].

Similarly, the velocity equation becomes

$$
\frac{d v_{\infty}}{d t}=\left(1-\frac{v_{\infty}^{2}}{w^{2}}\right)\left(w^{2} \frac{k}{L}-\frac{v_{\infty}}{\ell_{d}}\right) .
$$

Note that these are valid for any cosmological scenario We do expect the loop chopping efficiency $\tilde{c}$ to be a constant, regardless of the cosmological model, since it is supposed to be reflecting a rather deep and fundamental property of the evolution of a network. Indeed, we think that whether or not one finds a constant chopping efficiency can in some sense be seen as a measure of how accurately the analytic modelling is reproducing the true dynamics of the network.

We can now re-examine the question of the existence of 'scale invariant' attractor solutions. Again, scaling solutions of the form $L \propto t, L \propto H^{-1}$ or $L \propto d_{H}$, together with $v_{\infty}=$ const. will only exist provided one has

$$
a(t) \propto t^{\beta}, \quad \beta=\text { const. }, \quad 0<\beta<1,
$$

but now we also require

$$
\Omega=\text { const. }
$$

The simplest example of the second condition is of course a flat, $\Omega_{\mathrm{M} 0}=1$ universe, but there are examples of cosmological models which have attractors other than $\Omega=1$ [26]. In any case, note that there can be additional relations between the values of $\beta$ and $\Omega$ for specific models. Writing $L=\gamma t$ as before, the scaling solution is now given in the implicit form

$$
\gamma^{2}=w^{2} \frac{k(k+\tilde{c})}{4 \beta(1-\beta)}, \quad v^{2}=w^{2} \frac{k(1-\beta)}{\beta(k+\tilde{c})},
$$

where $k$ is (implicitly) the constant value of $k(v)$ for the appropriate value of velocity, and

$$
w=\frac{2(1-\beta)}{(1-\Omega) \beta k(k+\tilde{c})}\left[\left(1+\frac{(1-\Omega) \beta k(k+\tilde{c})}{(1-\beta)}\right)^{1 / 2}-1\right] .
$$

Again, although it may not be immediately obvious, it can be checked numerically that this solution is well-behaved for all sensible values of the parameters. If the two conditions above do not hold, then a scaling solution will not exist.

We should also mention another cosmologically important solution: in an open universe with $\Omega \rightarrow 0$, $a \propto t$, the asymptotic solution is

$$
\begin{gathered}
L=A t(\ln t)^{1 / 2} \quad A=\left[\frac{k_{n r} \tilde{c}}{2\left(1-k_{n r}\right)}\right]^{1 / 2} \sim 2.13 \tilde{c}^{1 / 2}, \\
v_{\infty}=B(\ln t)^{-1 / 2}, \quad B=\left[\frac{k_{n r}\left(1-k_{n r}\right)}{2 \tilde{c}}\right]^{1 / 2} \sim 0.21 \tilde{c}^{-1 / 2},
\end{gathered}
$$

with $k_{n r}$ given by(3.8). Note that this is not a scale-invariant solution, since $H^{-1}=t$ and $d_{H}=t \ln t$. In other words, by looking at the network one would be able to determine when the curvature-dominated period had started.

\footnotetext{
${ }^{1}$ There are some additional subtleties involved when discussing the mechanism of loop production in the case of Minkowski space string networks, which make it quite different from any cosmological scenario. We shall discuss this important point elsewhere [16].
} 


\section{DISCUSSION AND CONCLUSIONS}

In this paper we have presented a modified version of the velocity-dependent one-scale (VOS) model [1, 2, 18, 3 , which depends on a single free parameter, the loop chopping efficiency $\tilde{c}$. We have tested it against the largest and most accurate numerical simulations to date [15,16], and we find that it provides a good fit to the large-scale scaling properties of the string network in both the radiation and matter epochs, as well as in the transition between the two eras - we will describe these tests elsewhere [5]. These facts and its intrinsic simplicity make this model particularly suited for any analytic or semi-analytic study of cosmic strings where one is only interested in the large-scale features of the network.

We have re-analysed some simple evolutionary properties of cosmic string networks in the light of the VOS model and corresponding numerical simulations. An important conclusion to note is that any realistic cosmic string network is not scaling at any time from just before the epoch of equal matter and radiation through to the present day. This is something that must be properly taken into account particularly when discussing string-seeded structure formation scenarios with GUT-scale strings. The extended VOS model is also valid when deviations from scaling are even larger at late times in a universe which becomes dominated by curvature or a cosmological constant.

Finally, we considered the effects of radiation back-reaction on the scaling properties of the long string network, and we have shown that although the existence (or otherwise) of a scale-invariant attractor solution will not be affected, the quantitative scaling properties can be. In some cases, the suppression of string density can be quite dramatic (as we saw for small-scale global string simulations), although the string velocities always remain relativistic. For the most part, however, the density of a cosmic string network, whether local or global is only affected slightly by radiation backreaction effects.

Despite the many virtues of the VOS model, we are aware, of course, that the small number of available degrees of freedom means that this model is unable to provide a proper description of the small-scale properties of the network; these are important in a number of cosmological scenarios (and sometimes even crucial). Nevertheless, we believe that the phenomenological parameter $k$ does encode some important small-scale structure effects, though clearly a more detailed analytic and numerical study is still required. A number of possible approaches to the problem of string small-scale structure have been suggested in the literature 12.13], and our own analysis using Carter's elastic string model [24] will be discussed in a forthcoming publication [14].

\section{ACKNOWLEDGMENTS}

We would like to thank Pedro Avelino, Brandon Carter, Jonathan Moore, Levon Pogosian, Tanmay Vachaspati and Proty Wu for useful conversations. C.M. also acknowledges discussions with a number of participants in the EC Summer School 'Multi-fractals - Mathematics and Applications', held at the Isaac Newton Institute. C.M. is funded by FCT (Portugal) under 'Programa PRAXIS XXI' (grant no. PRAXIS XXI/BPD/11769/97).

This work was performed on COSMOS, the Origin2000 owned by the UK Computational Cosmology Consortium, supported by Silicon Graphics/Cray Research, HEFCE and PPARC.

[1] C.J.A.P. Martins and E.P.S. Shellard, Phys. Rev. D53, 575 (1996).

[2] C.J.A.P. Martins and E.P.S. Shellard, Phys. Rev. D54, 2535 (1996).

[3] C.J.A.P. Martins, Quantitative String Evolution, Ph.D. Thesis, University of Cambridge (1997).

[4] A. Vilenkin and E. P. S. Shellard, Cosmic Strings and other Topological Defects, (Cambridge University Press: Cambridge, 1994).

[5] C.J.A.P. Martins, E.P.S. Shellard and B. Allen, 'Testing the Velocity-dependent One-scale String Evolution Model', submitted to Phys. Rev. D (2000).

[6] A. Albrecht and N. Turok, Phys. Rev. D40, 973 (1989).

[7] D. Bennett and F. Bouchet, Phys. Rev. D41, 2408 (1990).

[8] B. Allen and E.P.S. Shellard, Phys. Rev. Lett. 64, 19 (1990).

[9] T.W.B. Kibble, Nucl. Phys. B252, 277 (1985).

[10] D.P. Bennett, Phys. Rev. D33, 872, Erratum D34 3932 (1986).

[11] B. Allen and R.R. Caldwell, Phys. Rev. Lett. 65, 1705 (1991);

B. Allen and R.R. Caldwell, Phys. Rev. D43, 2457 (1991); 
D. Austin, Phys. Rev. D48, 3422 (1993).

[12] F. Embacher, Nucl. Phys. B387, 129 (1992);

F. Embacher, Phys. Rev. D49, 5030 (1994).

[13] D. Austin, E.J. Copeland and T.W.B. Kibble, Phys. Rev. D48, 5594 (1993).

[14] C.J.A.P. Martins and E.P.S. Shellard, 'Wiggly cosmic string models', in preparation (2000).

[15] C.J.A.P. Martins, E.P.S. Shellard and B. Allen, 'The fractal properties of cosmic string networks', in preparation (2000).

[16] C.J.A.P. Martins, E.P.S. Shellard and B. Allen, 'High-resolution cosmic string simulations', in preparation (2000).

[17] C.J.A.P. Martins, Phys. Rev. D55, 5208 (1997).

[18] P.P. Avelino, R.R. Caldwell and C.J.A.P. Martins, Phys. Rev. D56, 4568 (19 97).

[19] P.P. Avelino, R.R. Caldwell and C.J.A.P. Martins, Phys. Rev. D59, 123509 (1999).

[20] C.J.A.P. Martins and E P.S. Shellard, Phys. Rev. D57, 7155 (1998).

[21] C.J.A.P. Martins and E P.S. Shellard, Phys. Lett. B445, 43 (1998).

[22] C. van de Bruck, Phys. Rev. D57, 1306 (1998).

[23] R.A. Battye \& E.P.S. Shellard, Phys. Rev. D53, 1811 (1996) .

[24] B. Carter, in The Formation and Evolution of Cosmic Strings, G.W. Gibbons et al. (eds.), Cambridge University Press(1990);

B. Carter, in Formation and Interactions of Topological Defects, A.C. Dav is \& R.H. Brandenberger (eds.), Plenum Press (1995);

B. Carter, in Tlaxcala lecture notes, 2nd Mexican School on Gravitation and Mathematical Physics, A. Garcia et al. (eds.) (1996). (see also hep-t h/9705172);

B. Carter, Phys.Rev. D60, 083502 (1999).

[25] M. Yamaguchi, Phys. Rev. D60, 103511 (1999);

M. Yamaguchi, J. Yokoyama and M. Kawasaki, Phys. Rev. D61, 061301 (2000).

[26] J.D. Barrow and J. Magueijo, Class. Quant. Grav. 16, 1435 (1999);

J.D. Barrow and J. Magueijo, Phys. Lett. B447, 246 (1999). 

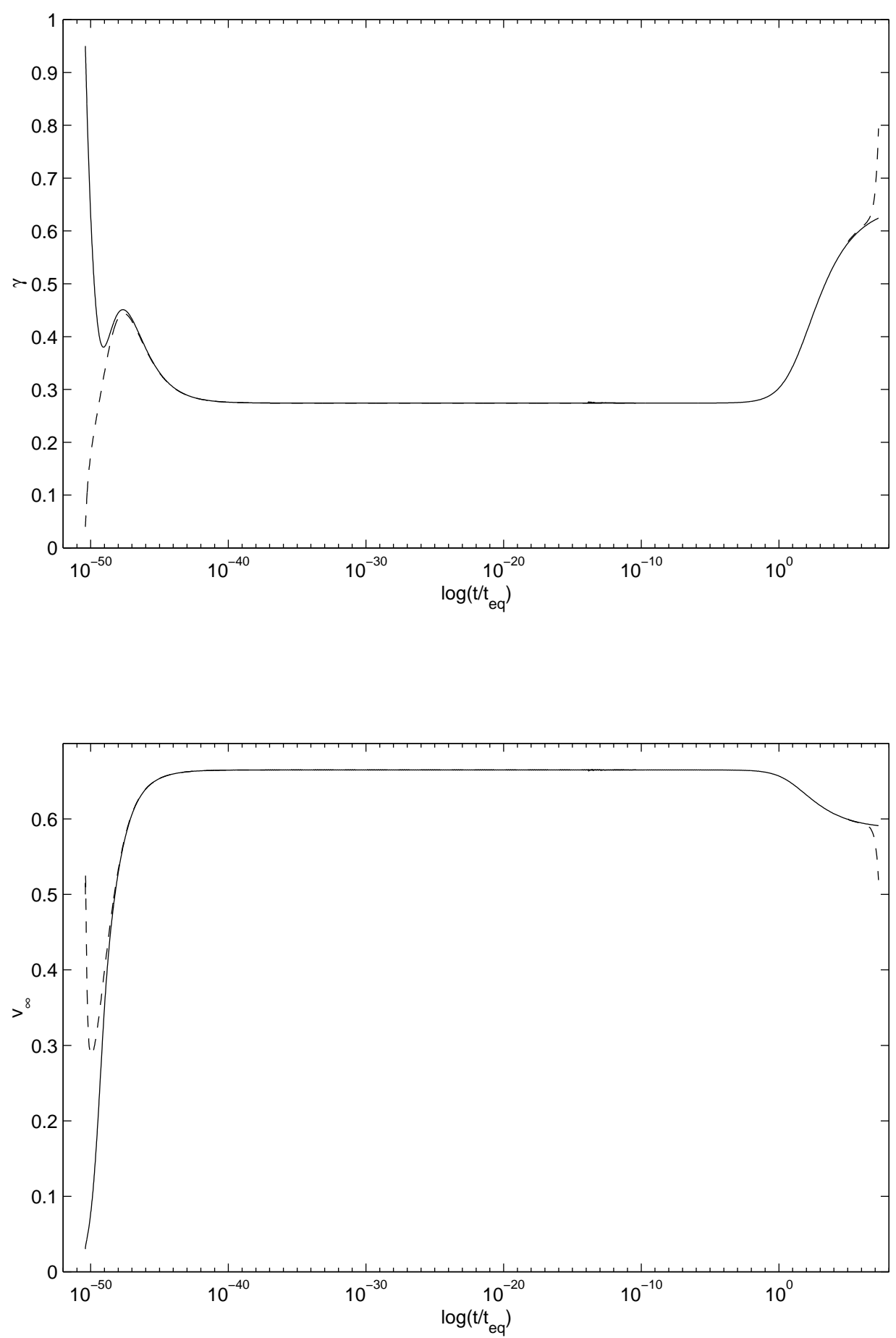

FIG. 1. The complete cosmological history of a GUT-scale cosmic string network. The evolution of $\gamma \equiv L / t$ is shown in the top panel, while the bottom one shows the network's RMS velocity $v_{\infty}$. Time is plotted relative to the epoch of equal matter and radiation densities; the plots start at the epoch of string formation and end at the present day. At early times, the solid curve corresponds to initial conditions typical of a first-order phase transition, while the dashed one corresponds to a second-order transition. At late times the solid curve corresponds to a model with $\Omega_{m}=1$, while the dashed one is for the observationally preferred case $\Omega_{m}=0.2, \Omega_{\Lambda}=0.8$ (to be discussed below). Note the deviations to the scaling behaviour. 

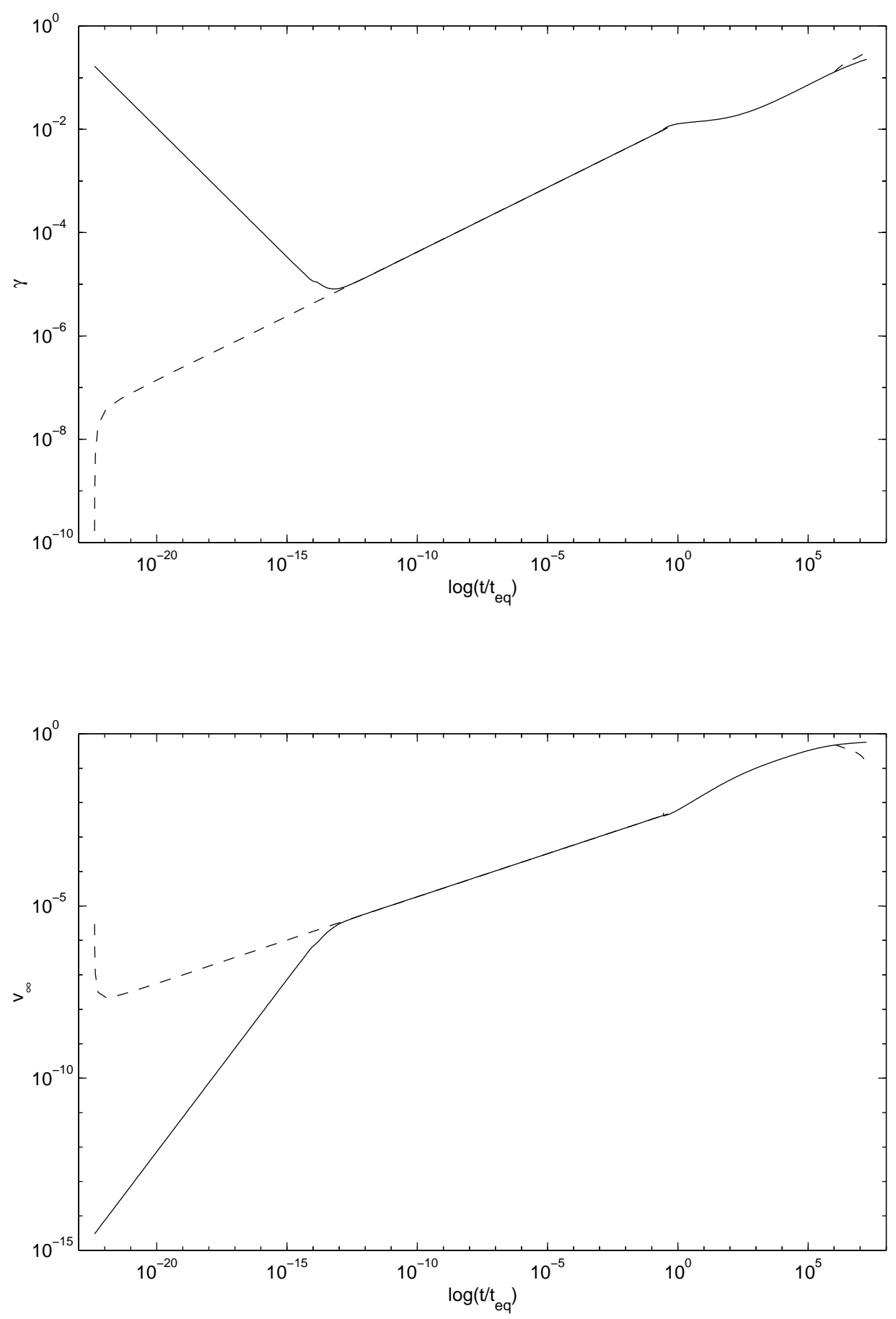

FIG. 2. Same as Fig. 1, but for an electroweak-scale cosmic string network. 

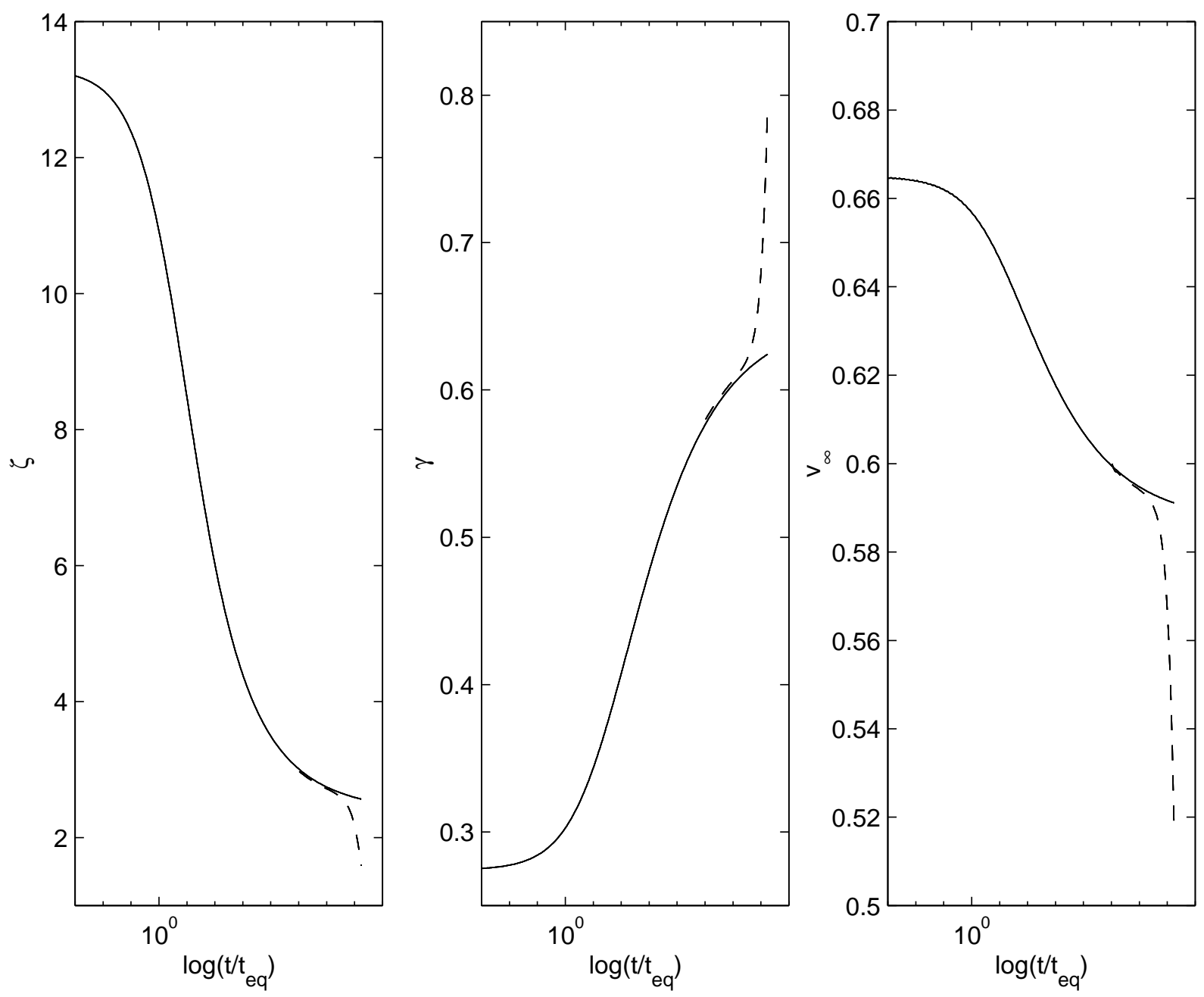

FIG. 3. A close-upof Fig. 1, showing the recent evolution of the GUT-scale string network. Also shown is parameter $\zeta$, a measure of the long-string density. Notice the dramatic chages once the cosmological constant dominates. 


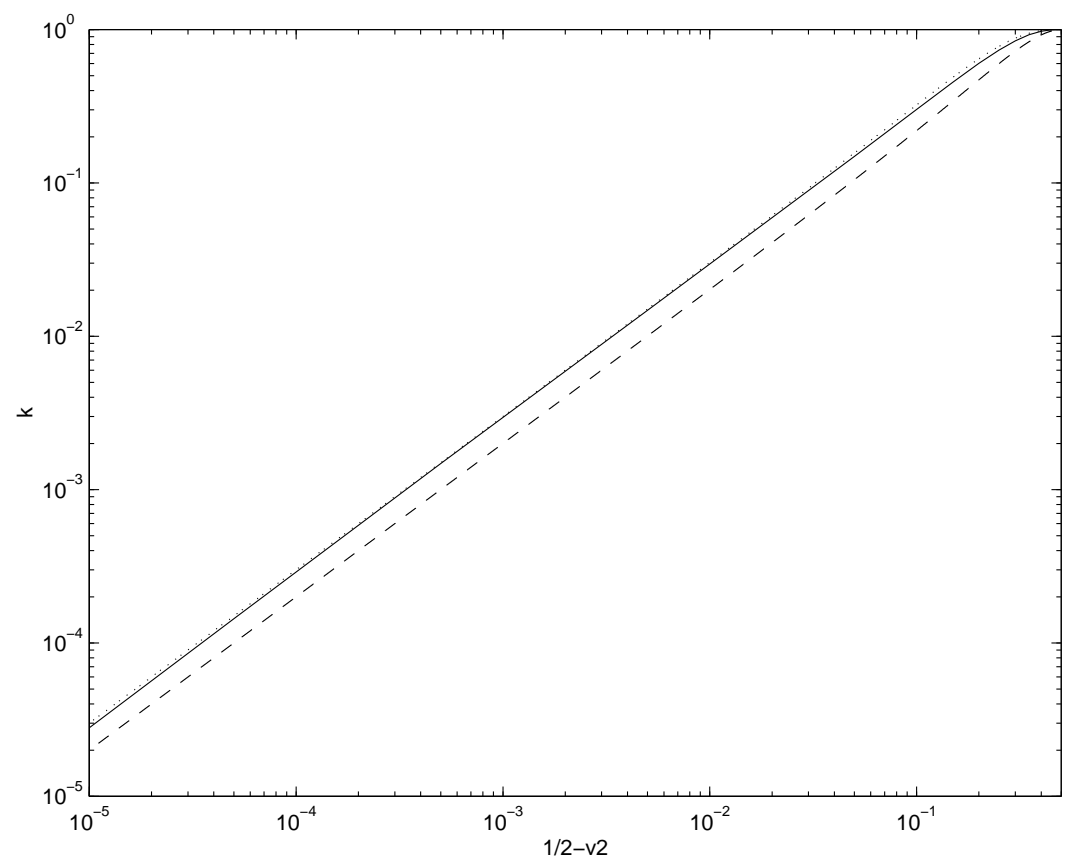

FIG. 4. Testing the ansatz (3.1) for $k(v)$ in the relativistic limit. The solid line corresponds to our numerical calculation of $\mathrm{k}$ for the helicoidal string solution, while the dashed and dotted lines correspond to the ansatz (3.1) with $\alpha=2$ and $\alpha=3$ respectively.
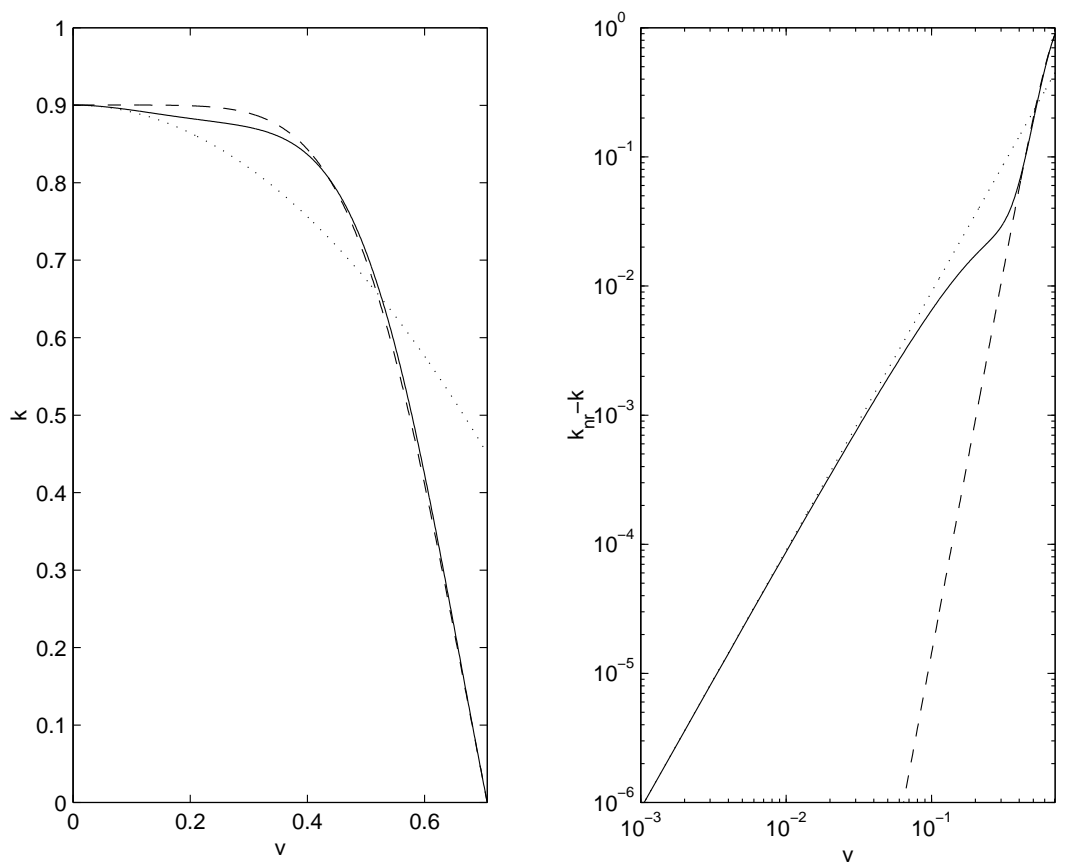

FIG. 5. Comparing our full ansatz for the momentum parameter (3.9) (solid line), and the simpler expressions for the relativistic and friction dominated regimes, (3.10) and (3.11) (dashed and dotted, respectively), for relativistic (left panel) and non-relativistic (right panel) velocities 

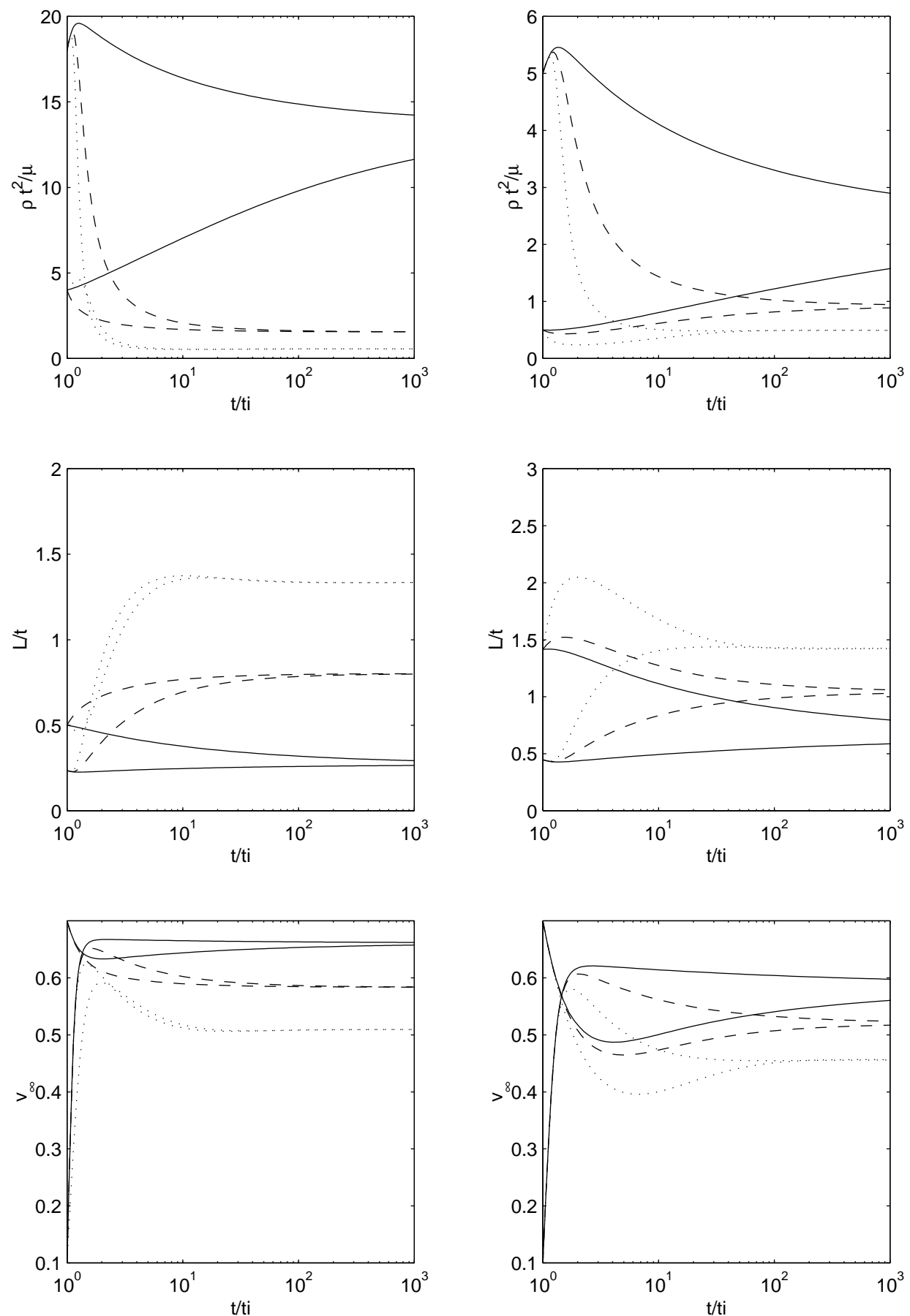

FIG. 6. The effect of gravitational back-reaction on the approach to scaling of a GUT-scale cosmic string network. Left-side panels correspond to the radiation era, while right-side ones are for the matter era. All plots have $\tilde{c}=0.23$; the back-reaction parameter is respectively $\Sigma=0$ (solid curves), $\Sigma=10$ (dashed) and $\Sigma=50$ (dotted). For each case two curves are plotted, corresponding to initial conditions typical of a first-order (low density and velocity) or second-order (high density and velocity) phase transition. The effect of friction due to particle scattering has been neglected, in order to mimic currently existing numerical simulations. 